\title{
Modelo de Simulación para evaluar las decisiones estratégicas de un comité local de AIESEC basados en el Balanced Scorecard
}

Leydi Yamile González Castillo, Diana Paola Hernández Rodríguez², José Ignacio Palacios Osma ${ }^{3}$

yamile637@hotmail.com,piohero2@gmail.com,jpalacios@udistrital.edu.co

${ }^{1}$ Grupo de Investigación en Comercio Electrónico en Colombia - GICOECOL, Universidad Francisco José de Caldas, Bogotá, Colombia.

${ }^{2}$ Grupo de Investigación en Comercio Electrónico en Colombia - GICOECOL, Universidad Francisco José de Caldas, Bogotá, Colombia

${ }^{3}$ Universidad Francisco José de Caldas - Facultad de Ingeniería, Bogotá, Colombia

DOI: 10.17013/risti.25.82-97

Resumen: Se desarrolla una propuesta que pretende ayudar a las juntas ejecutivas de cualquier comité local de AIESEC para poder evaluar sus planes anuales, así como las decisiones estratégicas más importantes en el desarrollo de su gestión. Para ello se integran dos herramientas a nivel organizacional que son el Balanced Scorecard (BSC) y la dinámica de sistemas. El BSC como herramienta que permite a cada integrante de la organización tener claro los objetivos y las actividades que permitirán el cumplimiento de una planeación propuesta y la dinámica de sistemas como herramienta que permitirá que el BSC pueda ser analizado de una forma flexible. Se emplea la metodología de la dinámica de sistemas de manera que las decisiones pueden ser simuladas y analizadas bajo una perspectiva más cercana a la realidad, obteniendo un modelo de simulación aceptado como una herramienta útil para la toma de decisiones estratégicas para un comité local de AIESEC.

Palabras-clave: Balanced Scorecard; dinámica de sistemas; toma de decisiones; simulación; diagramas causa-efecto.

\section{Model of simulation to Evaluate Strategic Decisions in an AIESEC local committee based on the balanced scorecard}

Abstract: A proposal is developed that will help the executive boards of any AIESEC local committee to evaluate their annual plans as well of the most important strategic decisions in the development of their management. For that are integrate two tools in organizational level such as Balanced Scorecard (BSC) and the dynamics of systems. The BSC as the tool that allows each member of the organization to clearly understand the objectives and activities that allow the fulfillment of a proposed planning and the dynamics of systems as the tool that may the BSC can be analyzed in a flexible way. It is use dynamics system's methodology 
in a way that decisions can be simulated and analyzed under a perspective closer to reality, obtaining an accepted simulation model as a useful tool for strategic decision making for a local AIESEC committee.

Keywords: Balanced Scorecard; dynamics of systems; decision making; simulation; diagram cause-effect.

\section{Introducción}

Una organización es un concepto que hace referencia a un conjunto de recursos que forman una estructura sistemática de relaciones de interacción, con el fin de lograr un objetivo distintivo que es su misión; para esto dentro de sus estructuras hay personas encargadas de definir y desarrollar la visión, la misión y la estrategia, lo cual debe ser compartido en todos los niveles para que puedan ser entendidas y apropiadas de la mejor manera, pues con base en los resultados obtenidos es que los directivos tomarán las decisiones estratégicas necesarias para dirigir las acciones al cumplimiento tanto de la visión como de la misión y estrategia definidas.

Dentro de las múltiples herramientas que ayudan en la toma de decisiones dentro de una organización se encuentra el Balanced Scorecard, que permite traducir la estrategia de la organización en objetivos concretos que pueden ser medidos, los cuales pueden ser tanto financieros como no financieros; sin embargo las relaciones que presenta esta herramienta por ser en una sola vía dificultan la toma de decisiones al no poder percibir a su debido tiempo la manera en la que influyen las variables en un proceso de realimentación.

Por lo anterior, se ve la necesidad de emplear una metodología que admita la construcción de modelos de simulación de sistemas complejos que permita evaluar las estrategias y cursos de acción previo a su implementación, pues al tomar decisiones basados en modelos rígidos en donde se realiza la medición y evaluación de las decisiones tomadas hasta después de cierto tiempo de implementación, genera el riesgo de tomar decisiones basados en resultados parciales de decisiones anteriores.

El objetivo del proyecto es construir un modelo de simulación empleando la metodología de dinámica de sistemas, que permita evaluar las decisiones estratégicas que implementará una junta ejecutiva de un comité local de AIESEC al comienzo de su gestión basado en la herramienta del Balanced Scorecard, ya que son las juntas ejecutivas las encargadas de plantear las metas y el plan de trabajo que se llevará a cabo en el comité local durante su año de gestión de acuerdo al propósito de la organización.

\section{Contextualización de AIESEC}

AIESEC en una organización sin ánimo de lucro creada para romper las barreras culturales que existían después de la segunda guerra mundial, actualmente se encuentra presente en más de 122 países y territorios.

$\mathrm{Su}$ visión es "paz y pleno desarrollo del potencial humano", para trabajar por ella hay seis valores fundamentales que son: la activación de liderazgo, demostrar integridad, vivir la diversidad, disfrutar la participación, buscar la excelencia, y actuar de forma 
sostenible, llevándose a cabo esta visión a través de oportunidades de manejo de equipo e intercambios internacionales.

AIESEC Internacional es el organismo que supervisa que los comités miembros están alcanzando su objetivo o visión, en cada país existen un comité miembro (MC - Member Committee) que son las juntas nacionales encargadas de supervisar el desarrollo de AIESEC en su territorio, y en un nivel básico, la forma en que AIESEC realiza experiencias de liderazgo a través de intercambios internacionales es por medio de los comités locales (LC - Local Committee), dentro de estos comités existe la figura de un presidente y unos vicepresidentes denominados Junta Ejecutiva (EB - Executive Board), quienes son los encargados de la toma de decisiones estratégicas dentro del comité local y son electos por los miembros del comité cada año.

\subsection{Procesos Internos}

Existe un proceso básico que realizan los EP (Exchange Participant) para tener su experiencia de intercambio denominado Customer Flow. El proceso inicia cuando una persona se entera de AIESEC y las oportunidades que ofrece, se interesa y decide registrarse en la plataforma e iniciar la búsqueda de la oportunidad adecuada para realizar su experiencia de intercambio.

Posteriormente, cuando el aplicante es aceptado en una oportunidad se denomina Match (MA), en ese momento el EP al realiza el pago a AIESEC por el acceso a la plataforma y la asesoría brindada, actualiza y gestiona los documentos que requiere para viajar, por último se genera la Realización (RE), proceso en el cual el EP viaja e inicia el proyecto o la práctica.

Sin embargo, al interior de la organización el proceso varía de acuerdo al programa, es decir, si son experiencias de intercambios entrantes (ICX) compuestas por las áreas IGIP (Incoming Global Intership Program) y IGCDP (Incoming Global Community Development Program), o experiencias de intercambios salientes (OGX) compuestas por las áreas OGIP (Outgoing Global Intership Program) y OGCDP (Outgoing Global Community Development Program); por lo que un comité local puede realizar mínimo un programa y máximo los cuatro.

\section{Marco Teórico}

\subsection{Planeación Estratégica}

La planeación estratégica no es un tema nuevo, sin embargo, en el Siglo XX se enfocó a las organizaciones debido a la necesidad de planear, que surge de los cambios derivados del proceso de globalización, formando parte del proceso administrativo mediante el cual se establecen directrices, se definen estrategias y se seleccionan alternativas y cursos de acción en función de objetivos y metas generales (H. Mintzberg \& B. Quinn, 1995).

Para realizar la planeación estratégica se deben tener en cuenta cuatro elementos:

El primero de ellos el contexto, que hace referencia al ambiente interno y externo en el cual se encuentra la organización y como éste influye en la misma, para ello sirve responder las preguntas ¿Quiénes somos? ¿Qué hacemos y para quienes? y ¿Dónde estamos?, 
El segundo elemento es el proceso de formulación estratégica, consiste en la forma como son pensadas, concebidas, comparadas y seleccionadas las estrategias; se compone de tres elementos lógicos, la intención estratégica correspondiente al propósito de la organización, la evaluación estratégica correspondiente a la evaluación total del contexto y la elección de la estrategia correspondiente a decidir qué acción tomar y como tomarla, para esto se responde a la pregunta ¿Dónde queremos ir y que resultados queremos lograr?;

El tercer es el contenido de la estrategia, lo cual consiste en la estrategia en si misma siguiendo con la pregunta ¿Cómo llegaremos?; y por cuarto y último se encuentra el proceso de implementación de la estrategia con la pregunta ¿Cómo mediremos el desempeño logrado? (J. H. M. García,2010) (M. Armijo \& G. Pública, 2009).

\subsection{Toma de decisiones}

La toma de decisiones es un proceso que se desarrolla en cada nivel de la organización con el fin de cumplir metas u objetivos establecidos, sin embargo no todas las decisiones son iguales ni producen las mismas consecuencias, dentro de los tipos de decisiones más comunes son las que se encuentran relacionadas con la posición jerárquica o el nivel administrativo ocupado por el o las personas que deciden, dentro de estas tenemos (L. A. R. Tovar, 2007):

- Decisiones estratégicas: Definidas, estructuradas y proyectadas a cumplir con los objetivos estratégicos, ligadas al desarrollo de la misión y la visión enfocadas en factores externos que afectan el crecimiento. Son decisiones de gran trascendencia pues involucran a toda la organización y son tomadas por los más altos ejecutivos de la pirámide jerárquica.

- Decisiones tácticas: Partiendo de los lineamientos sugeridos por las decisiones estratégicas son generadas por directivos intermedios y desarrolladas para las principales áreas de la organización que cumplirán con metas fijadas en lapsos de tiempo no muy largos, en caso de ser decisiones equivocadas no afectaran drásticamente la organización a menos que sean muy frecuentes.

- Decisiones operativas: Son tomadas por los ejecutivos que se sitúan en el nivel más bajo de la pirámide jerárquica y ejecutada por los niveles más bajos de la organización, relacionados con la asignación de tareas específicas, de rutina y procedimientos automáticos, sus implicaciones dentro de la organización son mínimos.

La toma de decisiones es el centro de la planeación debido a que la selección de entre varias alternativas determina el curso de acción de la organización, teniendo en cuenta que "todas las decisiones se toman en un ambiente de al menos una incertidumbre, que incluye la interacción de varias variables importantes donde hay ciertos riesgos involucrados al tomar decisiones" (E. Castillo, L. Verdes \& Y. I. C. Gastelúm, 2002) es necesario contar con un profundo conocimiento, una amplia experiencia en el tema para lograr el manejo de la información y el monitoreo del desempeño de la estrategia, para este proceso se han desarrollado y utilizado diferentes herramientas que paulatinamente han sido aceptadas y en algunos casos mejoradas como el BSC entre otras. 


\subsection{Balanced Scorecard (BSC)}

Es una herramienta para gestionar la estrategia a corto, mediano y largo plazo, desarrollada por Robert Kaplan y David Norton en 1990, que permite definir la estrategia de la organización al conceptualizar la misión, la visión y los valores, mediante la identificación y selección de los principales factores implicados en la misma y su conexión causal (J.L. Retolaza, J.T. Pruñonosa \& L. San-Jose, 2012).

El éxito del BSC depende de la comprensión de la estrategia de la empresa, pues esta herramienta a través de la relación coherente entre sus elementos, simplifica la gestión, prioriza lo importante, promueve el aprendizaje y puede motivar mejoras innovadoras en áreas tan críticas como productos, procesos, el cliente y el desarrollo del mercado (R. S. Kaplan \& D. P. Norton, 2004).

Una vez definida la estrategia principal de la organización, esta se traslada en objetivos concretos, denominados objetivos estratégicos, que serán evaluados a través de indicadores, dado que el BSC parte de la premisa que no se puede controlar lo que no se puede medir. Los objetivos estratégicos muestran aquello que se quiere conseguir; aunque lo más importante para la mayoría de las empresas es la rentabilidad, no es lo único, por lo que deben ser tomadas en cuenta las perspectivas del cliente, de los procesos internos, de formación y crecimiento, los cuales se pueden visualizar en un mapa estratégico. De manera que se garantiza que el modelo es equilibrado y no se centra únicamente en la rentabilidad presente, sino en aspectos no financieros claves para conseguir una rentabilidad futura (A. Fernández, 2001).

EL BSC aunque propone perspectivas para la evaluación de la estrategia, no explica cabalmente la realidad, pues las organizaciones son dinámicas e intervienen tiempos de retardo, procesos de realimentación y no tiene en cuenta que la relación entre las variables no es lineal sino curva, por lo que no propone una metodología que establezca y valide cada una de las relaciones causa-efecto y su impacto en la organización (D. M. Castañeda Olmos, 2012)

\subsection{Dinámica de Sistemas}

Es una metodología que estudia el comportamiento de los sistemas complejos, analizando la forma en que interactúan sus partes mediante la construcción de un modelo, teniendo en cuenta los flujos entre variables, los procesos de realimentación y las demoras, siendo muy útil para el estudio de fenómenos sociales ya que en estos se encuentran gran cantidad de elementos interrelacionados, pudiendo simularlos utilizando software computacional como herramienta para representar como las políticas, decisiones, retrasos y estructuras se interrelacionan en el tiempo para influir sobre el crecimiento y la estabilidad del sistema.

Dado que la dinámica de sistemas proporciona elementos conceptuales y metodológicos que permiten analizar los posibles efectos de decisiones a tomar en cuanto a su repercusión sobre el sistema en el mediano plazo y considera los retrasos entre la toma de decisión y el efecto de la misma (H. Zamorano, 2004), es una metodología muy útil para superar las limitaciones que ha presentado el BSC, incluso Kaplan y Norton "creen que la simulación con Dinámica de Sistemas será la expresión última de la estrategia 
de una organización y la base perfecta para el BSC (Norton citado en Rydzak, F; et.al, 2004)" (D. M. Castañeda Olmos, 2012).

La metodología de dinámica de sistemas, por ser un modelo sistémico no está predeterminado por un modelo matemático previo, sino por el establecimiento del análisis del sistema basado en el modelo mental de las personas que poseen el conocimiento del mismo, por lo que se dispone de unas fases específicas para su construcción y análisis, que son, fase de análisis, fase de modelado cualitativo o causal, fase de modelado cuantitativo, fase de simulación y fase de validación, por las que no se pasa de una forma progresiva y única sino que se puede ir de una fase a otra sin ningún orden especial cuantas veces sea necesario.

\section{Metodología}

El desarrollo del estudio se basa en la metodología de dinámica de sistemas, se inicia con la fase de análisis en donde se revisa la misión, la visión y se estudia el BSC de acuerdo con la realidad actual de los comités locales; realizando ajustes de acuerdo con la información suministrada en entrevistas a miembros de la organización, la experiencia e información sobre el BSC con la que se cuenta. Posteriormente se identifican los elementos que componen el sistema, sus relaciones y los tipos de influencia entre estos, realizando el diagrama causal del sistema aplicando lo descrito en la fase de modelado cualitativo o causal.

Partiendo del diagrama causal y del conocimiento del funcionamiento del sistema, se desarrolla el diagrama de Forrester seguido de la fase de simulación para terminar con la fase de validación, la cual se realiza simulando diferentes decisiones y verificando los resultados.

\subsection{Fase de análisis}

Se realiza una modificación del BSC debido a que del análisis realizado se evidencio que no se encuentra lo suficientemente alineado a la realidad de los comités locales, pues varios de los objetivos estratégicos e indicadores están más enfocados a las labores a realizar a nivel nacional o internacional que a nivel local.

Por lo anterior se plantea un Balanced Scorecard para ser base del modelo de simulación para evaluar las decisiones estratégicas de un comité local de la siguiente manera:

- Perspectiva principal "Qué hacemos", enfocada en la medición de las experiencias de liderazgo a través de los diferentes programas de intercambio y de la experiencia de membresía. Sus objetivos estratégicos son generar mayor cantidad de experiencias de intercambio sociales, profesionales, de membresía y aumentar la satisfacción de las experiencias.

- Perspectiva aprendizaje y capacidad, hace referencia a la cantidad de recurso humano, su desarrollo y la cantidad de recursos físicos al interior de un comité local para cumplir los objetivos planteados en la perspectiva principal. Sus objetivos estratégicos son aumentar la capacidad del talento humano, desarrollar el talento humano y aumentar la cantidad de activos fijos. 
- Perspectiva de procesos internos, la cual hace referencia a los procesos críticos que influyen en la consecución de los objetivos estratégicos planteados en la perspectiva principal. Sus objetivos estratégicos son asegurar la legalidad de las experiencias de intercambio, divulgar adecuadamente la información, analizar e investigar el mercado, vender la oportunidad indicada de la manera correcta y tener eficiencia operativa.

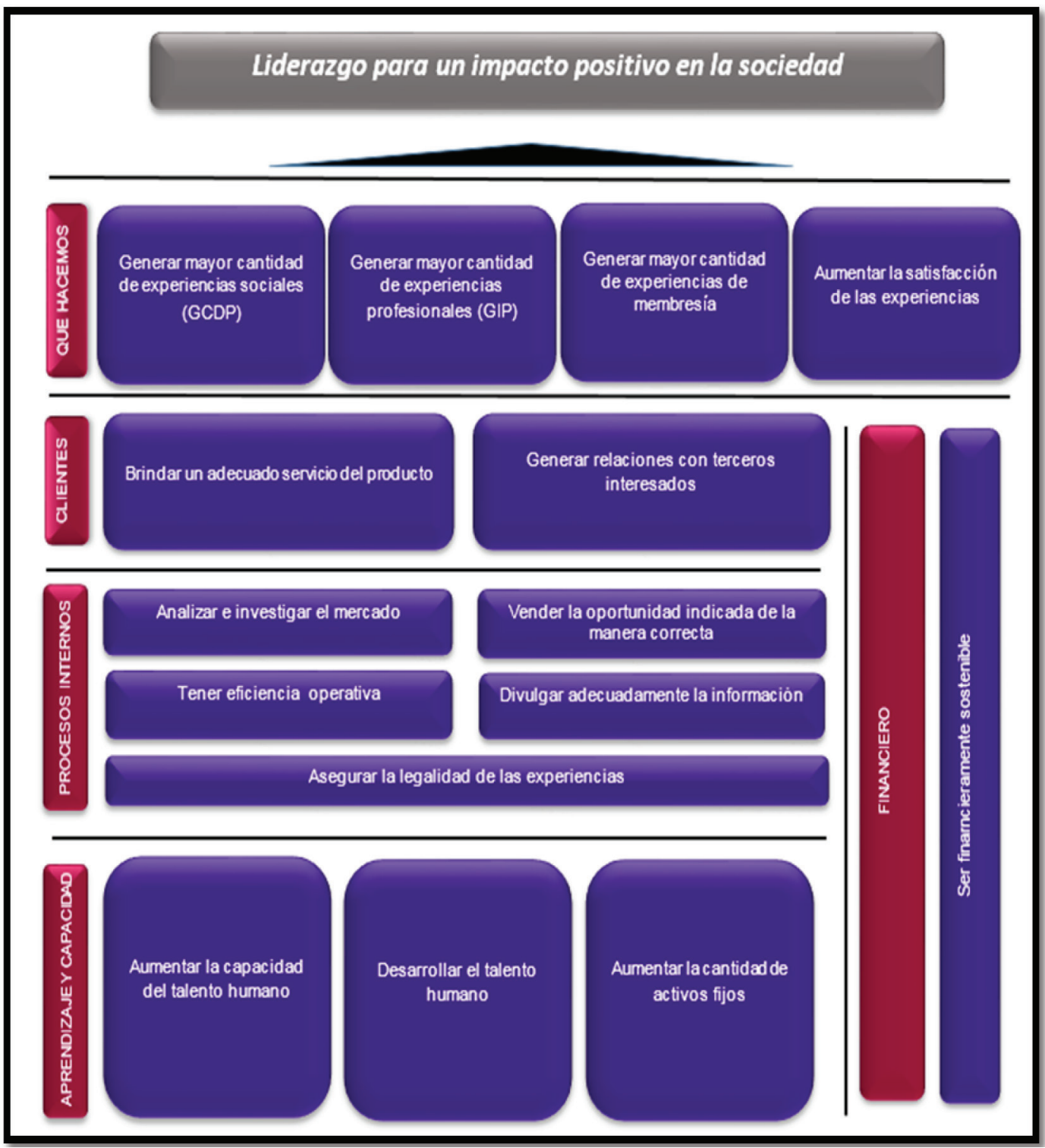

Figura 1 - Mapa estratégico del Balanced Scorecard propuesto. 
- Perspectiva de clientes, hace referencia a las relaciones con los stakeholders, con quienes se desarrollan vínculos o alianzas que permiten o facilitan la generación de las experiencias de liderazgo. Los objetivos estratégicos son generar relaciones con terceros interesados y brindar un adecuado servicio del producto.

- Perspectiva financiera, enfocada a la administración de los recursos financieros para soportar las demás perspectivas, por lo que esta perspectiva será transversal en el Balanced Scorecard. Su objetivo estratégico es ser financieramente sostenible, haciendo referencia a ser sostenibles al tener un control de las inversiones y/o gastos realizados de manera que los ingresos sean mayores a los egresos.

Es importante tener en cuenta que las metas por cada indicador deberán ser planteadas por cada comité local de acuerdo a su realidad y plan estratégico planteado, pues la medición y control de estos indicadores los ayudará a tomar decisiones para cumplir con lo planteado para su gestión.

De manera gráfica se puede observar el mapa estratégico del Balanced Scorecard propuesto en la figura 1.

\subsection{Fase de modelado cualitativo o causal}

El diagrama causal propuesto para realizar el modelo de simulación se puede observar en la figura 2, este diagrama contempla el funcionamiento básico de un comité local, evidenciando los procesos básicos de membresía, experiencias e inversiones que se encuentran inmersos en el comportamiento dinámico del sistema.

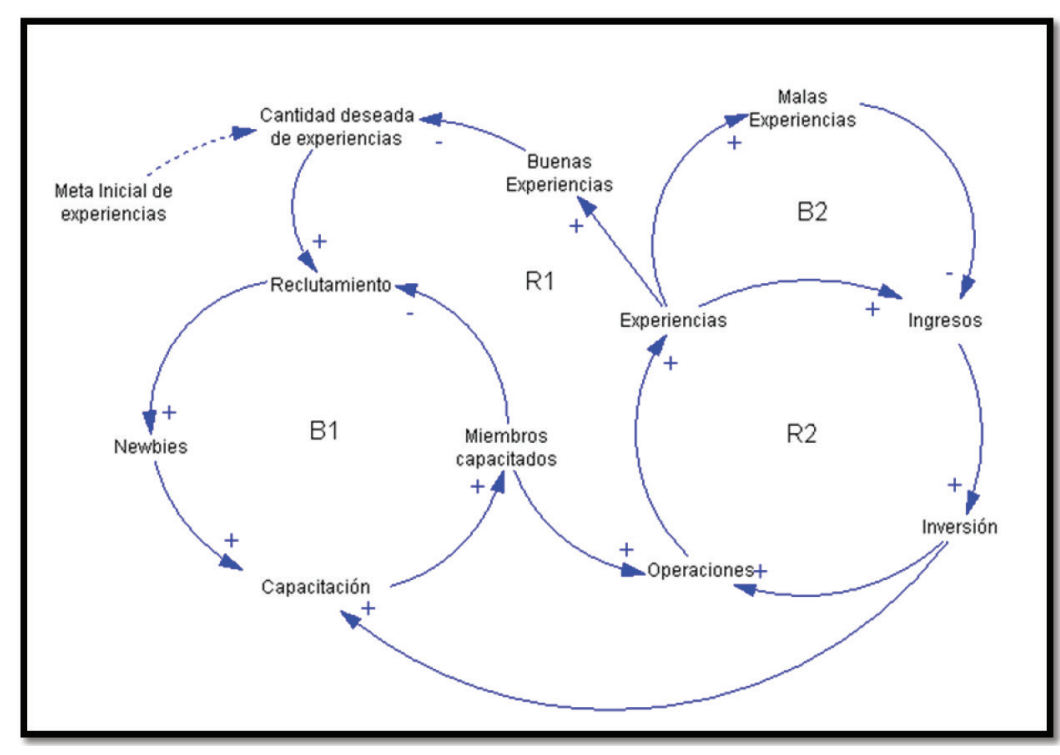

Figura 2 - Diagrama causal. 
La dinámica del sistema inicia con el proceso de membresía (B1), con la necesidad percibida de incorporar miembros al comité denominada reclutamiento, que surge de la cantidad de experiencias que se desean brindar, siendo la meta planteada al inicio de la gestión por la junta ejecutiva local, que hace referencia a la perspectiva principal, pues se enfoca en los resultados que se quieren obtener.

Con el reclutamiento se incorporan newbies, los cuales deben ser capacitados para poder realizar las operaciones de la manera adecuada generando que mientras mayor cantidad de miembros capacitados se tenga menor es la necesidad de reclutamiento, haciendo referencia a la perspectiva aprendizaje y capacidad a todo el proceso de capacitación y desarrollo de la membresía.

La variable operaciones dentro de este diagrama hace referencia a la perspectiva de clientes, pues es donde permite la apertura de oportunidades para las experiencias de ICX; teniendo en cuenta esto, se puede observar el proceso de experiencias (R1), el cual inicia con miembros capacitados que pueden realizar mayor cantidad de operaciones las cuales generan una mayor cantidad de experiencias pudiendo ser buenas o malas, siendo este proceso la representación de la perspectiva de procesos internos, pues al generar una mayor cantidad de experiencias buenas se disminuye la cantidad de experiencias que se desean generar.

Para poder realizar los dos procesos anteriores se debe llevar a cabo el proceso de inversiones (B2, R2) siendo la representación de la perspectiva financiera, la cual se genera cuando se realizan experiencias pues cada una genera ingresos, dependiendo el programa es el monto, al tener ingresos se pueden realizar inversiones en operaciones o en capacitaciones dependiendo de la necesidad, por otro lado se puede evidenciar que al generar malas experiencias se produce una disminución de los ingresos debido a que se deberá indemnizar a la persona que tuvo esta mala experiencia de intercambio.

\subsection{Fase de modelado cuantitativo}

De acuerdo al diagrama causal y teniendo en cuenta cada una de las perspectivas del Balanced Scorecard se desarrolla el diagrama de Forrester, de manera que se realiza una representación aproximada del sistema real que permite la evaluación de las decisiones estratégicas que toma una junta ejecutiva de un comité local.

El diagrama de Forrester se divide en cuatro módulos correspondientes a las perspectivas del Balanced Scorecard propuesto para un comité local de AIESEC, expresando como flujos, niveles y tasas auxiliares las variables del diagrama causal, como se puede evidenciar en el modelo del sistema en la figura 3 .

El primer módulo es la representación de la perspectiva aprendizaje y capacidad del BSC; esta perspectiva cuenta con los parámetros de reclutamiento, newbies, tasa de deserción, inversión en capacitación y unidades de capacitación por \$ invertido; con las variables de nivel miembros y capacitaciones de miembros; y con las variables auxiliares deserción, capacitaciones y pérdida de conocimiento.

El segundo módulo es la representación de la perspectiva clientes del BSC, esta perspectiva cuenta con elementos provenientes de la perspectiva anterior de miembros y promedio de conocimiento; los parámetros de posibles ventas por $\$$, inversiones IGIP 
(Área de Intercambios Entrantes Profesionales) y IGCDP (Área de Intercambios Entrantes Sociales), miembros IGIP y IGCDP, tasa efectividad ventas IGIP y IGCDP; con las variables de nivel citas IGIP y IGCDP, pago IGIP; y con las variables auxiliares operaciones IGIP y IGCDP, pérdidas de citas IGIP y IGCDP, ventas IGIP y IGCDP, ingresos IGIP1.

El tercera módulo es la representación de la perspectiva procesos internos del BSC, esta perspectiva cuenta con elementos provenientes de la perspectiva anterior de miembros y promedio de conocimiento; los parámetros de tasa MA (Match) RE (Realización) IGIP y IGCDP, inversiones OGCDP (Área de Intercambios Salientes Sociales) y OGIP (Área de Intercambios Salientes Profesionales), miembros OGCDP y OGIP, cantidad de personas por $\$$, tasa de conversión IP a MA OGCDP y OGIP, tasa de conversión MA a RE OGCDP y OGIP; con las variables de nivel oportunidades IGIP y IGCDP, oportunidades machadas IGIP y IGCDP, pago IGIP, delivery IGIP y IGCDP, buenas realizaciones IGIP y IGCDP, pago malas realizaciones IGIP y IGCDP, malas realizaciones IGIP y IGCDP, aplicantes OGCDP y OGIP, pago match OGCDP y OGIP, machado OGCDP y OGIP, buenas realizaciones OGCDP y OGIP, pago malas realizaciones OGCDP y OGIP, malas realizaciones OGCDP y OGIP; y con las variables auxiliares paso pago IGIP, malas XP, búsqueda de EPs (Exchange Participant) y de EPs IGCDP, delivery, delivery pago, ingresos IGIP y IGCDP, tiempo pagos, delivery IGIP y IGCDP, delivery 1 y 2 , delivery IGIP 2, egresos malas XP IGIP y IGCDP, Pago malas XP IGIP, delivery ICX, EPs OGCDP y OGIP, aplicantes perdidos, búsqueda oportunidades y búsqueda oportunidades2, ingresos OGCDP y OGIP, paso pago match y paso pago match oGIP, asesorìa OGCDP y OGIP, asesorìa OGCDP 2 y OGIP 2, egresos malas XP OGCDP y OGIP, pagos OGCDP y OGIP, perdida de aplicantes.

El cuarto módulo es la representación de la perspectiva financiera del BSC, esta perspectiva cuenta con las variables auxiliares ingresos, egresos, entrada invoice, salida invoice, pago invoice, contador; y con las variables de nivel flujo de caja, acumulación de ingresos.

Los parámetros representan todos aquellos valores o constantes que son particulares y conocidos en cada comité bien sea por su comportamiento histórico o su estado actual que pueden ser modificables por el usuario del programa, algunas de ellas por medio de la interfaz, las variables auxiliares varían instantáneamente en respuesta al comportamiento de las variables de nivel que son aquellas que van acumulando los resultados de acciones tomadas anteriormente.

\subsection{Fase de simulación}

Esta fase contempla un horizonte temporal de 12 meses, se asignan magnitudes a los parámetros y ecuaciones a las variables auxiliares definidas en el diagrama de Forrester por medio del software de simulación iThink, el cual es auto-generador de código, brindando facilidad en la elaboración de ecuaciones y permitiendo la experimentación con el sistema.

De esta manera se generan las ecuaciones matemáticas que definen el comportamiento del sistema las cuales serán un sistema de ecuaciones diferenciales que generalmente no se pueden solucionar analíticamente, por lo debe ser simulado en un computador, proporcionando un entorno donde se realizan las pruebas de los modelos mentales que se tienen de la realidad a través del uso de la simulación. 


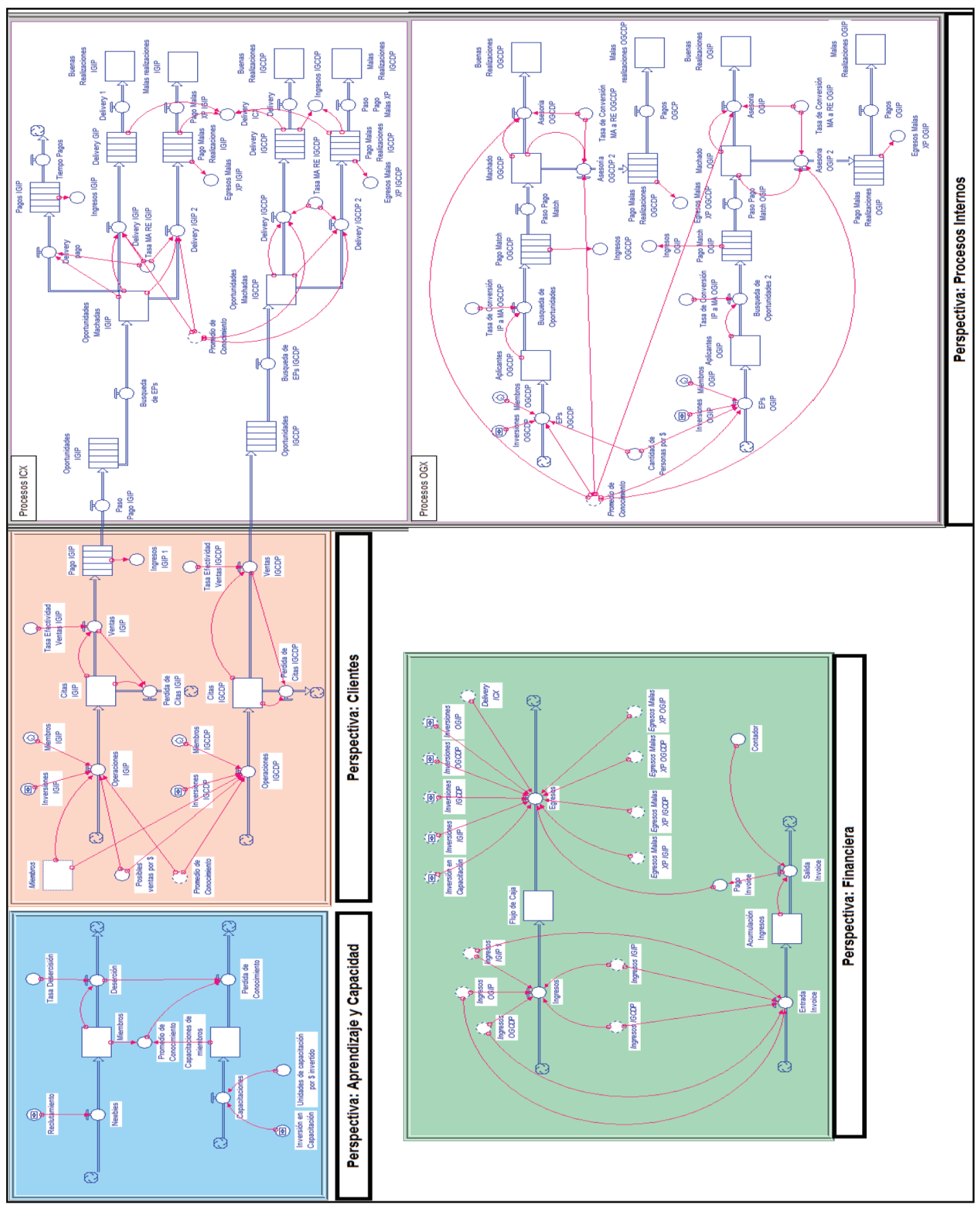

Figura 3 - Perspectivas del modelo del sistema. 


\subsection{Fase de validación}

Para realizar la validación de resultados del modelo de simulación realizado, se contemplan tres entidades locales para evaluar las decisiones tomadas durante un año de gestión. Teniendo en cuenta que AIESEC en Colombia cuenta con treinta (30) entidades locales de diferentes tamaños que aportan a los resultados en diversas proporciones, estos se dividieron en tres grupos de diez entidades cada uno de acuerdo al porcentaje de participación que obtuvieron en los resultados del año, eligiendo a aquellas que representarán a su grupo de acuerdo a los resultados y con mayor acceso a la información requerida para realizar la simulación.

Del primer grupo que cuenta con entidades cuya participación dentro de la organización varía entre el $11 \%$ a $4 \%$ siendo entidades grandes que manejan los cuatro (4) programas, se selecciona a AIESEC en Andes que cuenta con una participación de 11\%; del segundo grupo que cuenta con entidades cuya participación varía entre el $4 \%$ a $2 \%$, se selecciona a AIESEC en Javeriana que cuenta con una participación de 3\%; y del tercer grupo que cuenta con entidades cuya participación varía entre el $2 \%$ a $0 \%$, se selecciona a AIESEC en Cúcuta que cuenta con una participación de 1\% y maneja dos (2) de los cuatro (4) programas que realiza AIESEC.

Una vez simulada la información se comparan los resultados obtenidos con los presentados en la realidad, obtenido lo siguiente:

\section{AIESEC Andes}

$\mathrm{Al}$ realizar la simulación con los datos de la entidad local, después de los 12 meses de gestión y al compararlos con la cantidad de experiencias que en realidad se generaron, se obtuvieron los resultados de la Tabla 1 por programa, teniendo en cuenta que esta entidad maneja los cuatro programas de intercambio, OGCDP, OGIP, IGCDP e IGIP.

\begin{tabular}{llll}
\hline Programa & $\begin{array}{l}\text { Cantidad de intercambios } \\
\text { resultado de simulación }\end{array}$ & $\begin{array}{l}\text { Cantidad de intercambios } \\
\text { realizados }\end{array}$ & Diferencia absoluta \\
\hline$O G C D P$ & 193 & 176 & $17-10 \%$ \\
\hline$O G I P$ & 66 & 68 & $2-3 \%$ \\
\hline$I G C D P$ & 70 & 98 & $28-29 \%$ \\
\hline$I G I P$ & 22 & 26 & $4-15 \%$ \\
\hline
\end{tabular}

Tabla 1 - Comparación de resultados numéricos del comité Andes.

\section{AIESEC Javeriana}

Al realizar la simulación con los datos de la entidad local, después de los 12 meses de gestión y al compararlos con la cantidad de experiencias que en realidad se generaron, se obtuvieron los resultados de la tabla 2 por programa, teniendo en cuenta que esta entidad maneja los cuatro programas de intercambio, OGCDP, OGIP, IGCDP e IGIP. 


\begin{tabular}{llll}
\hline Programa & $\begin{array}{l}\text { Cantidad de intercambios } \\
\text { resultado de simulación }\end{array}$ & $\begin{array}{l}\text { Cantidad de } \\
\text { intercambios realizados }\end{array}$ & Diferencia absoluta \\
\hline OGCDP & 70 & 54 & $16-30 \%$ \\
\hline OGIP & 41 & 24 & $17-71 \%$ \\
\hline$I G C D P$ & 30 & 38 & $8-21 \%$ \\
\hline$I G I P$ & 10 & 15 & $5-33 \%$ \\
\hline
\end{tabular}

Tabla 2 - Comparación de resultados numéricos del comité Javeriana.

\section{AIESEC Cúcuta}

$\mathrm{Al}$ realizar la simulación con los datos de la entidad local, después de los 12 meses de gestión y al compararlos con la cantidad de experiencias que en realidad se generaron, se obtuvieron los resultados de la tabla 3 por programa, teniendo en cuenta que esta entidad maneja dos de los cuatro programas de intercambio, OGCDP e IGCDP.

Debido a que este comité sólo maneja dos programas tanto OGIP como IGIP no cuenta con destinación de recursos financieros ni humanos, reflejado en la no generación de experiencias de liderazgo.

\begin{tabular}{llll}
\hline Programa & $\begin{array}{l}\text { Cantidad de intercambios } \\
\text { resultado de simulación }\end{array}$ & $\begin{array}{l}\text { Cantidad de } \\
\text { intercambios realizados }\end{array}$ & Diferencia absoluta \\
\hline$O G C D P$ & 23 & 19 & $4-21 \%$ \\
\hline$I G C D P$ & 28 & 19 & $9-47 \%$ \\
\hline
\end{tabular}

Tabla 3 - Comparación de resultados numéricos del comité Cúcuta.

Después de haber simulado los tres comités y hallar las diferencias que hay entre los datos reales y los obtenidos por el modelo de simulación se calcula la magnitud del error relativo (ER) y error relativo medio (ERM), para garantizar que el modelo de simulación desarrollado representa coherentemente la realidad de AIESEC, y pueda ser aceptado como una herramienta útil para la toma de decisiones estratégicas.

De acuerdo a los resultados obtenidos, el modelo de simulación desarrollado es aceptado como una herramienta útil para la toma de decisiones estratégicas para un comité local de AIESEC al contar con una confiabilidad de aproximadamente el $75 \%$, debido a que actualmente, los comités locales presentan un promedio de error entre las planeaciones estratégicas planteadas y los resultados alcanzados mayor al $47 \%$, lo que hace de la herramienta un modelo confiable en comparación con los resultados actuales.

\section{Conclusiones}

El modelo de simulación consistió en realizar un Balance Scorecard dinámico con el cual se evalúan los resultados que pueden generar las estrategias que se toman dentro de los comités locales, a partir de esto concluimos que es necesario tener un seguimiento de la 
información más riguroso a algunos datos para seguir corroborando la funcionalidad de este modelo, y crear mejoras que disminuyan las diferencias con los resultados, como lo son las tasas de conversión y efectividad de ventas, en donde las estrategias pueden estar enfocadas en la disminución de estos valores y confirmar su efectividad de acuerdo a los cambios realizados.

Se evidencia que el Balanced Scorecard como herramienta administrativa de planeación es útil para cumplir con los objetivos inicialmente establecidos, sin embargo por tener una ruta rígida en una sola vía se dificulta la revisión anticipada de las posibles decisiones a tomar, lo cual se facilita con la dinámica de sistemas al ver a la estructura de una organización como un sistema que se interrelaciona y realimenta con información para la toma de decisiones de una manera integral.

La inclusión de la dinámica de sistemas en este modelo contribuye a entender las relaciones causa-efecto de las variables que hacen parte del Balanced Scorecard, de tal manera que se vislumbra cómo la variación de cada una de ellas puede afectar al sistema, y al ser susceptible de simulación se convierte en una herramienta de apoyo útil para evaluar las decisiones estratégicas en este caso principalmente de inversión, teniendo una visión más enfocada en resultados por cada una de las estrategias a implementar y mitigando la incertidumbre de su implementación, pues se cuenta con un estimado de resultados con anticipación a su implementación.

De manera general se percibe que al dinamizar el Balanced Scorecard se entienden mejor la relación entre las variables, permitiendo identificar factores claves que afectan en mayor medida el cumplimiento de los objetivos estratégicos de las organizaciones, lo cual es un gran soporte al momento de analizar estrategias para la toma de decisiones.

Se evidencia que la inversión económica tanto en los miembros como en cada una de las áreas influencia los resultados y se complementan, siendo más relevante la inversión realizada en capacitaciones para aumentar el promedio de conocimiento de la membresía, puesto que un miembro capacitado e involucrado, es un miembro empoderado que se enfocará en los resultados incluso con poca inversión en el área.

Es importante ratificar que este modelo no pretende pronosticas sino evaluar las decisiones estratégicas que se puedan tomar con respecto a cantidad de membresía de una comité local y su destinación para cada uno de los programas que maneja, así como las inversiones financieras a realizar y como estas se ven reflejadas en la generación de experiencias de intercambio, de acuerdo a la realidad de cada uno de los comités locales.

\section{Referencias}

AIESEC Colombia (2014). Politicas Financieras. Colombia: AIESEC en Colombia.

Aracil, J., \& Gordillo, F. (1995). Dinámica de sistemas. Madid: Isdefe.

Armijo, M. \& Pública, G. (2009). Manual de planificación estratégica e indicadores de desempeño en el sector público. ILPES/CEPAL. 
Asuaga, C., Cambeiro, P., Cami, M., \& Mouradian, I. (2007). Gestión de teatros públicos: Una adaptación del Cuadro de Mando Integral. Quantum: revista de administración, contabilidad y economía, 2, 93-111.

Carmen Muñoz, E. (2009). Cuadro de Mando Integral (Balanced Scorecard) para la gestión bibliotecaria: pautas para una aplicación. Investigación bibliotecológica, 23, 105-126.

Castañeda Olmos, D. M. (2012). Modelo tipo balanced scorecard dinámico como herramienta de la estrategia en ambientes dinámicos. Tesis Maestría en Administración, University of Medellín, Facultad de Ciencias Económicas y Administrativas.

Castillo, E., Verdes, L., \& Gastelúm, Y. I. C. (2002). Toma de decisiones, Un enfoque Particular. Trabajo de ascenso no publicado. UNET. San Cristóbal. Venezuela.

Fernández, A. (2001). El Balanced Scorecard. Revista de antiguos alumnos del IESE. 2001(Mar), 31-42.

García, J. H. M. (2010). La inteligencia de negocios como herramienta para la toma de decisiones estratégicas en las empresas. Análisis de su aplicabilidad en el contexto corporativo colombiano. Retrieved from: http://www.bdigital.unal.edu.co/3098/

Gross, H. (2014). Learn By Traveling Through AIESEC: The Importance of Studying and Experiencing Intercultural Communication. Degree Bachelor of Arts, California Polytechnic State University, San Luis Obispo.

Kaplan, R. S., \& Norton, D. P. (2004). Focusing your organization on strategy-with the balanced scorecard. Cambridge: Harvard Business School Publishing.

Mintzberg, H. (2006). Cinco Ps para estratégia, O processo da estratégia. Conceitos, contextos e casos selecionados (Cap. 1.1, pp. 24-29). Porto Alegre: Bookman.

Mintzberg, H., \& QUINN, B. (1995). Planeación estratégica. México: Prentice Hall.

Nario, O. L. C. J. P. (2009). Mapas Estratégicos y Cuadro de Mando Integral en la Implantación de la Estratégia. Modelo de Gestión por Competencias en el Desarrollo de una Organización Capaz. Tesis de grado, Facultad de Ciencias Económicas y de Administración, Universidad de la República, Uruguay.

Petul, H. A. Q. (2013). Planeación estratégica en la administración con enfoque a sistemas. Recuperado de https://www.gestiopolis.com/planeacion-estrategica-enla-administracion-con-enfoque-a-sistemas/

Retolaza, J. L., Pruñonosa, J. T., \& San-Jose, L. (2012). El cuadro de mando integral en las Entidades No Lucrativas. Revista de Contabilidad y Dirección, 14, 89-106.

Saldías Cerda, J. R. \& Chacur, A. A. (2006). Sistemas de control de gestion, analisis para organizaciones sin fines de lucro. Revista Ingeniería Industrial, 5.

Santa Catalina, I. M. (2010). Modelo de Dinámica de Sistemas para la implantación de Tecnologías de la Información en la Gestión Estratégica Universitaria. Tesis Doctor en Informática, Universidad del País Vasco, San Sebastián. 
Sarabia, Á. A. (1995). La teoría general de sistemas. Madrid: Isdefe.

Sterman, J. D. (2000). Business dynamics: systems thinking and modeling for a complex world (vol. 19). Boston: Irwin/McGraw-Hill.

Tovar, L. A. R. (2007). Mapa de las teorías de a organización: una orientación para empresas. Gaceta Ide@ s CONCYTEG, 2, 30.

Valderrama Salazar, P. A. (2013). Implementación del Balanced Scorecard para seguimiento del Conpes 3675 de 2010. Especializacion en administracion de empresas, Universidad EAN Bogotá D.C.

Vega Almeida, R. L. (2006). La relación dialógica entre la planificación estratégica y el aprendizaje organizacional. Acimed, 14(6), 6.

Zamorano, H. (2004). Modelos de simulación para la gestión del conocimiento y su medición en instituciones sin fines de lucro y dependencias gubernamentales. Retrieved from http://www.galleanoyzamorano.com.ar/museo.pdf 